\title{
Artemisinin derivatives prevent obesity by inducing browning of WAT and enhancing BAT function
}

Cell Research (2016) 26:1169-1172. doi:10.1038/cr.2016.108; published online 16 September 2016

\section{Dear Editor,}

Obesity and its related disorders have become a global pandemic [1]. Obesity leads to multiple chronic morbidities such as fatty liver, type 2 diabetes and cardiovascular diseases, and even increases the risk of several cancers. Five drugs have been approved by the Food and Drug Administration (FDA) for long-term weight management; however, there is still a long way to go before achieving satisfactory clinical outcome due to modest efficacy and unfavorable side effects [2]. Current anti-obesity medications focus mainly on restricting energy intake either by decreasing fat absorption or by suppressing appetite. There is no clinical record of successful pharmacotherapy to treat obesity by enhancing energy mobilization. Thermogenesis by brown adipose tissue (BAT) and beige adipose tissue which arises from the browning of white adipose tissue (WAT) is emerging as an appealing strategy to combat obesity in recent years. Thermogenesis improves metabolic homeostasis by dissipating energy in the form of heat. This process is achieved through the activation of uncoupling protein 1 (UCP1). UCP1 is unique to thermogenic adipocytes including brown and beige adipocytes, serving as a morphological and functional marker for these cells [3]. A few compounds have been reported to activate thermogenesis [4-6]. These compounds were found previously to elicit beneficial effects on metabolism and enhancing thermogenesis was discovered later as one of the mechanisms. Up to now, no high-throughput screening has been carried out to find small-molecule compounds able to activate thermogenesis for pharmacological applications such as treating obesity or other related metabolic disorders. With this purpose in mind, we established two high-throughput methods. 3T3-L1 cells are a well-characterized preadipocyte cell line, which differentiate into typical white adipocytes. During the differentiation of 3T3-L1 cells, expression of UCP1 is barely detectable [7]. We therefore reasoned that expression of UCP1 in 3T3L1 cells could serve as a readout for the browning of white adipocytes and this system could be used to iden- tify compounds that elicit thermogenesis. 3T3-L1 cells were seeded onto 96-well plates and then developed into mature adipocytes under standard differentiation condition in the presence or absence of various compounds $(10 \mu \mathrm{M})$. Oil Red O staining was used to eliminate compounds detrimental to adipogensis. Compounds that did not affect lipid accumulation were subjected to one-step qPCR to detect the expression of browning-related genes including UCP1 (Supplementary information, Figure S1A). In total, we screened over 3000 compounds and identified one candidate, artemether (ATM, chemical structure shown in Figure 1A). 3T3-L1 cells exposed to ATM were smaller in size and contained multiple small lipid droplets, which is the typical feature of thermogenic adipocytes (Supplementary information, Figure S1B). One-step qPCR showed that the relative mRNA levels of browning-related genes, such as PRDM16, PGCla and $U C P 1$, were elevated after treatment of ATM (Supplementary information, Figure S1C). We went on to confirm the browning effect of ATM in the C3H10T1/2 cells, which is a mesenchymal stem cell (MSC) line that can be induced to undergo adipogenic development. In vitro differentiation of $\mathrm{C} 3 \mathrm{H} 10 \mathrm{~T} 1 / 2$ cells can be divided into two phases: a commitment phase induced by BMP4 or BMP7 and a differentiation phase induced by a defined cocktail. BMP7 during the commitment phase drives C3H10T1/2 cells to develop into brown adipocytes [8], whereas BMP4 promotes adipogenesis [9]. We treated C3H10T1/2 cells with ATM throughout the BMP4-induced commitment phase and the differentiation phase (group b), or in the commitment phase only (group c), or in the differentiation phase only (group d; Supplementary information, Figure S1D). Adipocytes in groups $\mathrm{b}$ and d displayed browning features (Supplementary information, Figure S1E and S1F), demonstrating that ATM functions in the differentiation phase. ATM-treated C3H10T1/2 cells displayed the typical morphology of thermogenic adipocytes: smaller in size and containing plurilocular lipid droplets (Figure 1B). The expression levels of browning-related genes such as $U C P 1, P G C 1 \alpha$ and PRDM16, and mitochondrial gene cytochrome $C$ 
A

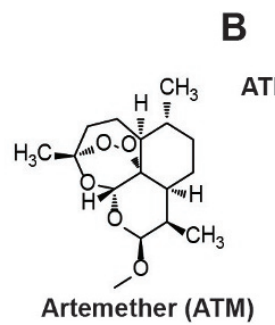

D

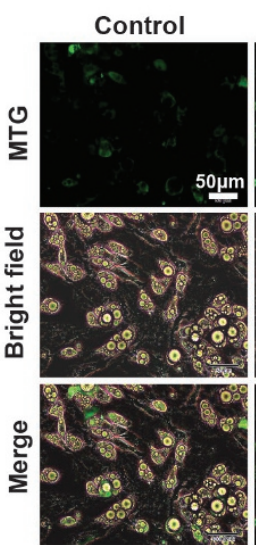

$B$

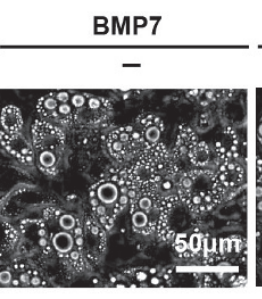

BMP4

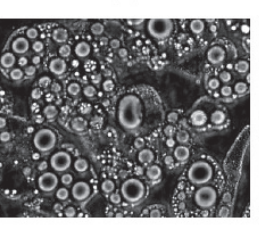

E

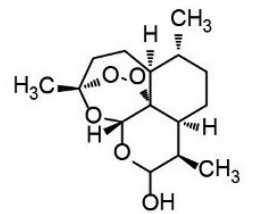

Dihydroartemisinin (DHA)

G

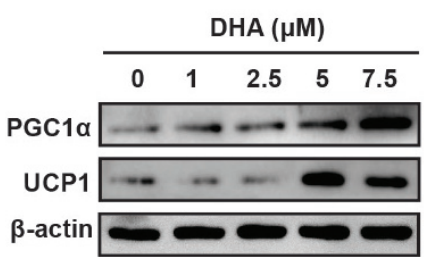

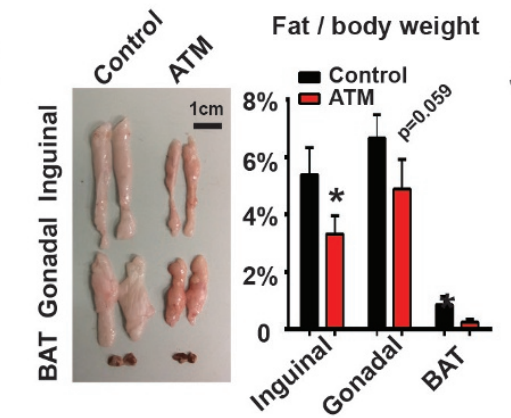

M

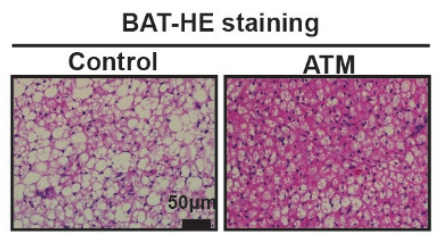

$\mathbf{N}$

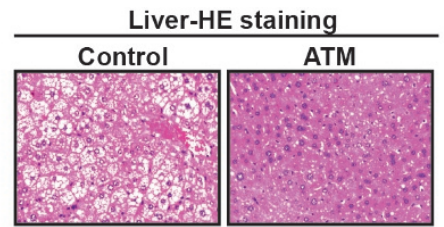

$\mathbf{Q}$

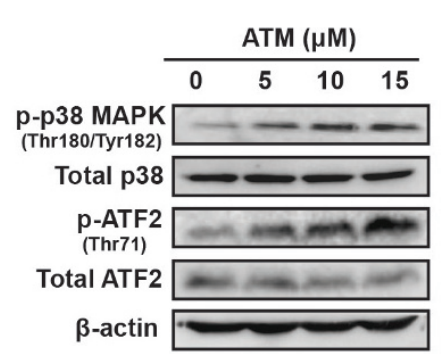

$\mathbf{J}$

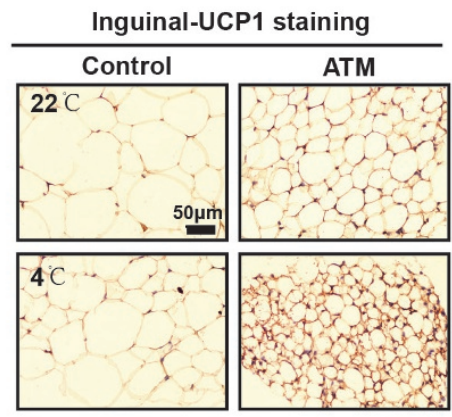

$\mathbf{F}$

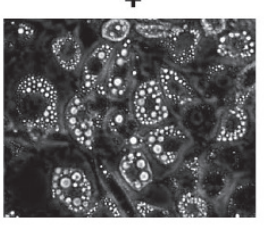

C

\begin{tabular}{|c|c|c|}
\hline \multicolumn{2}{|c|}{$\mathrm{BMP} 7$} & BMP4 \\
\hline ATM $(\mu \mathrm{M})$ & ) 00 & $\begin{array}{lll}5 & 10 & 15\end{array}$ \\
\hline PRDM16 [ & $6 \square$ & 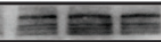 \\
\hline $\operatorname{PGC1\alpha }[$ & - & $\ldots-2$ \\
\hline UCP1 [ & $\because$ & $-2-1$ \\
\hline toc & 6 & $=$ \\
\hline & & \\
\hline
\end{tabular}

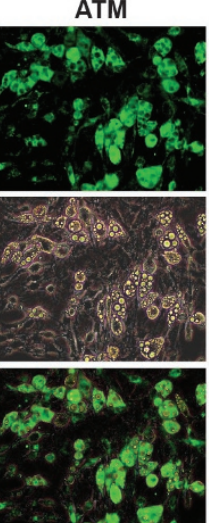

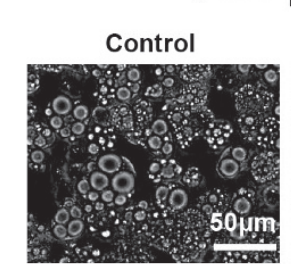

H

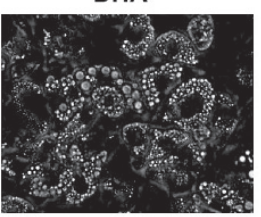

Body weight

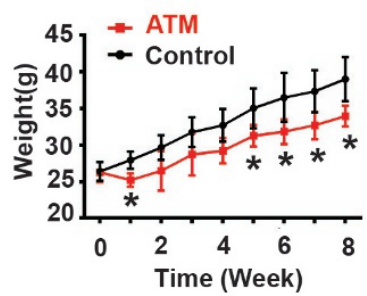

K

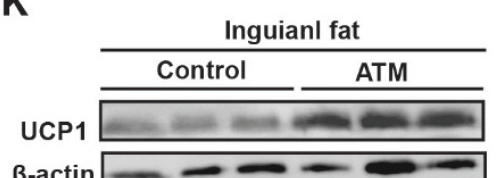

$\beta$-actin $\longrightarrow-\infty-\infty$
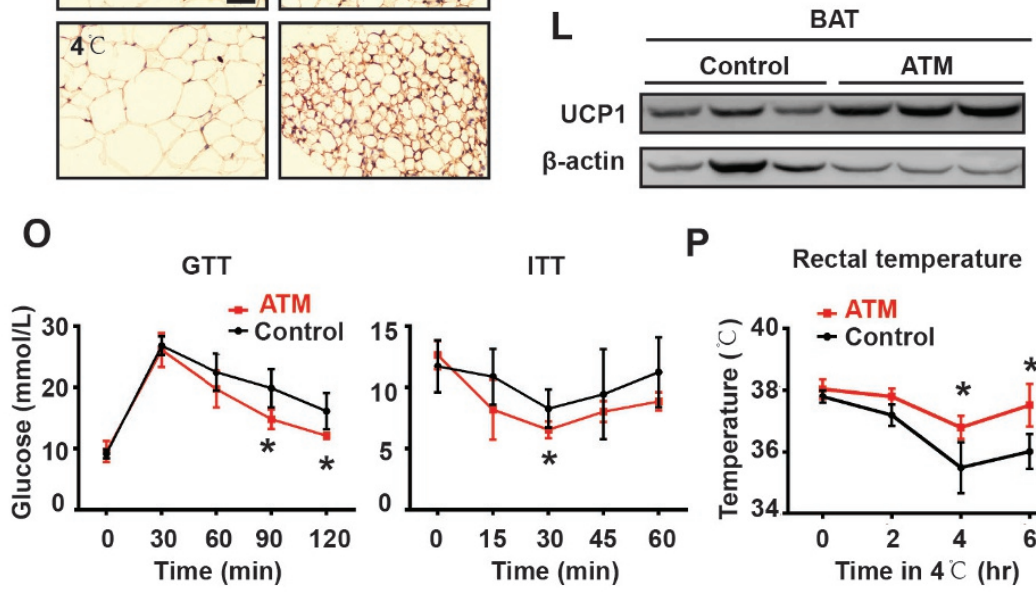

$\mathbf{P}$

Rectal temperature

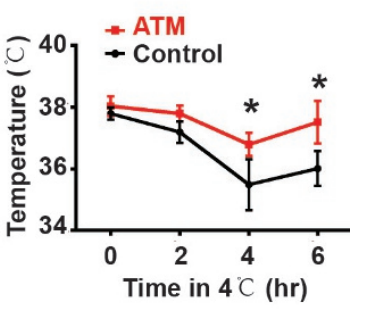

$\mathbf{R}$

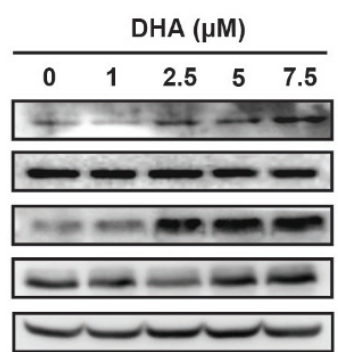

$\mathbf{S}$

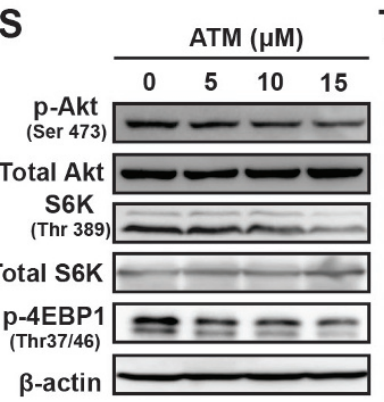

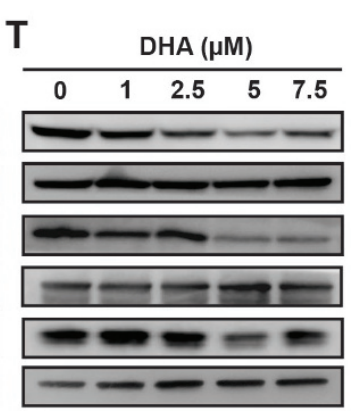


Figure 1 Artemisinin derivatives prevent obesity by inducing browning in WAT and enhancing thermogenesis in BAT. (A) Chemical structure of ATM. (B) The morphological change induced by ATM $(15 \mu \mathrm{M})$ in BMP4-committed C3H10T1/2 cells, with BMP7-committed cells as a positive control. (C) The increase in the expression levels of browning-related genes resulted from ATM treatment in BMP4-committed C3H10T1/2 cells, with BMP7-committed cells as a positive control. (D) MTG staining in BMP4-committed C3H10T1/2 cells treated with or without ATM $(15 \mu \mathrm{M})$. (E) The chemical structure of DHA. (F) The morphological change induced by DHA $(7.5 \mu \mathrm{M})$ in BMP4-committed C3H10T1/2 cells. (G) The increase in the expression levels of browning-related genes resulted from DHA treatment in BMP4-committed C3H10T1/2 cells. $(\mathbf{H})$ The body weigh of HFD-fed mice over treatment of ATM for 8 weeks. ${ }^{*} P<0.05$; student's $t$-test; $n=8$. (I) The morphology and weight ratio of adipose tissues of HFD-fed mice after treatment with ATM for 8 weeks. ${ }^{*} P<0.05$; student's $t$-test; $n=8$. (J) Representative IHC staining of UCP1 in the inguinal fat of HFD-fed mice after treatment with ATM for 8 weeks. (K-L) Western blotting analysis of UCP1 expression in the inguinal fat (K) and BAT (L) of HFD-fed mice after treatment with ATM for 8 weeks. (M-N) Representative HE staining of BAT (M) and the liver tissue (N) of HFD-fed mice treated with or without ATM for 8 weeks. (0) GTT and ITT of HFD-fed mice treated with or without ATM for 8 weeks. ${ }^{*} P<0.05$; student's $t$-test; $n$ $=5$. $(P)$ Rectal temperatures of HFD-fed mice exposed in cold environment for the indicated periods after being treated with or without ATM for 8 weeks. * $P<0.05$; student's $t$-test; $n=5$. (Q-T) Western blotting analyses of the indicated proteins in BMP4-committed C3H10T1/2 cells after treatment with ATM $(\mathbf{Q}, \mathbf{S})$ or DHA $(\mathbf{R}, \mathbf{T})$. In $\mathbf{B}-\mathbf{D}, \mathbf{F}, \mathbf{G}$, and $\mathbf{Q}-\mathbf{T}, \mathrm{C} 3 \mathrm{H} 10 \mathrm{~T} 1 / 2$ cells were treated with BMP4 or BMP7 for 3 days followed by incubation with the induction cocktail with or without ATM or DHA for another 8 days. The indicated assays were then performed.

(Cyto $C$ ) were upregulated by ATM in a dose-dependent manner (Figure 1C). Moreover, extensive mitochondrial biosynthesis was observed in ATM-treated C3H10T1/2 cells as shown by MitoTracker Green (MTG) staining (Figure 1D). To further verify the browning effect of ATM, stromal vascular fractions (SVFs) containing primary preadipocytes were isolated from the inguinal adipose tissues of male C57BL6/J mice. SVFs exposed to ATM developed into brown-like adipocytes characterized by plurilocular lipid droplets (Supplementary information, Figure S1G) and enhanced expression of browning-related genes (Supplementary information, Figure $\mathrm{S} 1 \mathrm{H})$. Taken together, ATM induces browning during the differentiation of white adipocytes. In contrast, among the previously identified thermogenesis-activating compounds, resveratrol [4] and salsalate [5] enhance the function of BAT, while berberine mainly promotes thermogenesis in mature brown and white adipocytes [6].

ATM is a first-line malaria medicine. Chemically, it is semi-synthetic derivative of artemisinin (also known as qinghaosu). Artemisinin-based therapy is currently the most effective treatment for uncomplicated Plasmodium falciparum malaria. Common derivatives of artemisinin include dihydroartemisinin (DHA, chemical feature shown in Figure 1E), ATM, artesunate and arteether. Among these, DHA is the metabolite of artemisinin in vivo [10]. To explore whether other derivatives of artemisinin have similar browning effect as ATM, we exposed $\mathrm{C} 3 \mathrm{H} 10 \mathrm{~T} 1 / 2$ cells to different doses of DHA. DHA induced browning-like features in $\mathrm{C} 3 \mathrm{H} 10 \mathrm{~T} 1 / 2$ cells as well, as illustrated by the smaller cell size, multiple lipid droplets (Figure 1F) and elevated expression levels of UCP1 and PGC1a (Figure 1G), suggesting that artemisinin and its derivatives share similar browning effect, which is also confirmed by the treatment with artemisinin, artesunate and arteether (data not shown).
To explore whether ATM is able to induce browning in vivo, we subcutaneously injected ATM into the inguinal fat pads of male C57BL6/J mice fed with a high fat diet (HFD). $15 \mu \mathrm{M}$ of ATM was injected subcutaneously into the inguinal fat pads of mice $(100 \mu l$ on each side, twice a week). Eight weeks after injection, ATM-treated mice showed lower body weight (Supplementary information, Figure S2A), less adipose tissues (Supplementary information, Figure S2B and S2C), better metabolism (Supplementary information, Figure S2D) and improved fatty liver (Supplementary information, Figure S2E) compared with the control group. Enhanced UCP1 expression in inguinal adipose tissue and better body temperature conservation capability were also observed in ATM-treated mice (Supplementary information, Figure S2F and S2G).

To further evaluate the pharmacological potential of ATM, we assessed the systematic effect of ATM through intraperitoneal (i.p.) injection in HFD-induced obese mice. ATM $(20 \mathrm{mg} / \mathrm{kg}$ body weight $)$ was given twice a week for 8 weeks. ATM significantly inhibited body weight gain induced by HFD (Figure 1H). Both the volume and weight of WAT and BAT decreased remarkably in ATM-treated mice (Figure 1I). Expression of UCP1, detected by immunohistochemistry (IHC) staining and western blotting in inguinal adipose tissues, was upregulated in mice housed either at room temperature or at $4{ }^{\circ} \mathrm{C}$ (Figure $1 \mathrm{~J}$ and $1 \mathrm{~K}$ ). Aside from the effects in WAT, ATM also induced UCP1 upregulation and morphological alteration in BAT (Figure 1L and $1 \mathrm{M}$ ), suggesting that enhanced thermogenesis in BAT may also contribute to weight loss in ATM-treated mice. Along with the decreased expansion of adipose tissues, fatty liver resulted from HFD was largely ameliorated after ATM treatment in comparison with the control group (Figure 1N). Insulin tolerance test (ITT) and glucose tolerance test (GTT) revealed significantly improved metabolism in 
ATM-treated mice (Figure 1O). Meanwhile, ATM-treated mice displayed better body temperature conservation when exposed to $4{ }^{\circ} \mathrm{C}$ (Figure 1P). Although ATM can protect mice from developing HFD-induced obesity, it did not affect the normal growth and metabolism of lean mice (Supplementary Information, Figure S2H-S2L). Notably, lean mice treated with ATM have smaller inguinal adipocytes (Supplementary information, Figure S2J) and accordingly gained better capability to preserve body temperature in cold environment (Supplementary information, Figure S2M).

We also explored the mechanism of ATM-induced browning. Consistent with previous findings that the p38 MAPK/ATF2 axis is indispensable for thermogenesis and the Akt/mTOR pathway is deactivated during browning [11], the p38 MAPK/ATF2 axis was activated and the Akt/mTOR pathway deactivated during browning of C3H10T1/2 cells induced by ATM or DHA (Figure 1Q$1 \mathrm{~T})$. This suggests that artemisinin derivatives may prevent obesity by inducing browning during adipogenesis through regulating these two pathways. The molecular target of artemisinin derivatives will be further characterized.

In summary, we unveil that artemisinin derivatives prevent obesity and improve obesity-related metabolic disorders in a rodent model. In vitro, in the presence of artemisinin derivatives, preadipocytes or MSCs acquire browning features during adipogenesis; in vivo, these derivatives induce browning in WAT and promote thermogenesis in BAT. Artemisinin, a gift from ancient Chinese medicine, has brought benefits to millions of malaria patients over decades. With cautious optimism, this compound and its derivatives would benefit more humans in the future.

\section{Acknowledgments}

This work was supported by the National Key Basic Research Project (2013CB530601) and the National Natural Science Foundation of China (the State Key Program, 31030048 and 81390350 to Qi-qun Tang). The department is supported by 985 Project 985 III-YFX0302.

Ping Lu ${ }^{1}$, Fu-chuang Zhang ${ }^{2}$, Shu-wen Qian ${ }^{1}$, $\mathrm{Xi} \mathrm{Li}{ }^{1}$, Zhao-meng Cui ${ }^{1}$, Yong-jun Dang ${ }^{1}$, Qi-qun Tang ${ }^{1,2}$

${ }^{I}$ Key Laboratory of Metabolism and Molecular Medicine, the Ministry of Education; Department of Biochemistry and Molecular Biology, Fudan University Shanghai Medical College, Shanghai 200032, China; ${ }^{2}$ Institute of Stem Cell Research and Regenerative Medicine, Institutes of Biomedical Sciences, Fudan University, Shanghai 200032, China

Correspondence: Qi-qun Tang ${ }^{\mathrm{a}}$, Yong-jun Dang ${ }^{\mathrm{b}}$

${ }^{a}$ Tel: 86-21-54237198

E-mail: qqtang@shmu.edu

b-mail: yongjundang@fudan.edu.cn

\section{References}

1 NCD Risk Factor Collaboration (NCD-RisC). Lancet 2016; 387:13771396.

2 Patel D. Metabolism 2015; 64:1376-1385.

3 Pfeifer A, Hoffmann LS. Аппи Rev Pharmacol Toxicol 2015; 55:207227.

4 Andrade JM, Frade AC, Guimaraes JB, et al. Eur J Nutr 2014; 53:1503-1510.

5 van Dam AD, Nahon KJ, Kooijman S, et al. Diabetes 2015; 64:15441554.

6 Zhang Z, Zhang H, Li B, et al. Nat Commun 2014; 5:5493.

7 Tang QQ, Lane MD. Annu Rev Biochem 2012; 81:715-736.

8 Tseng YH, Kokkotou E, Schulz TJ, et al. Nature 2008; 454:10001004.

9 Tang QQ, Otto TC, Lane MD. Proc Natl Acad Sci USA 2004; 101:9607-9611.

10 Klayman DL. Science 1985; 228:1049-1055.

11 Cai H, Dong LQ, Liu F. Trends Pharmacol Sci 2016; 37:303-317.

(Supplementary information is linked to the online version of the paper on the Cell Research website.) 